Sección uno: Ensayo

Mujer y Educación. Un largo camino hacia la igualdad

\title{
Estudiantes universitarias conversando sobre violencia de género. Resultados preliminares ${ }^{1}$
}

University students talking about gender violence. Preliminary results

\author{
Violeta Luque-Ribelles \\ Departamento de Psicología \\ Universidad de Cádiz \\ violeta.luque@uca.es \\ María Escalona \\ Fundación Don Bosco Salesianos \\ Social \\ ps.mariaescalona@gmail.com
}

\section{Resumen}

Tras entrar en vigor la Ley 1/2004 de Medidas de Protección Integral contra la Violencia de Género (VG), se han identificado progresos, pero también transformaciones en algunos discursos que obstaculizan los avances en igualdad entre mujeres y hombres (Lorente, 2007). Por un lado, las investigaciones exponen que los mitos en torno a la VG siguen vigentes (Bosch y Ferrer, 2012; Bosch, Ferrer y Navarro, 2013; Delegación del Gobierno contra la Violencia de Género, 2014). Por otro, la Universidad debía incluir la igualdad de género y no discriminación transversalmente en la formación, docencia e investigación. Sin embargo, la incorporación en la formación de grado ha sido de bajo impacto y superficial. Este trabajo muestra las ideas que las universitarias de un Grado en Psicología tienen de la VG. Se realizaron dos grupos focales en los que participaron catorce estudiantes. Los resultados giran en torno a cinco categorías: sistema patriarcal, concepto de VG, causas de la VG, tipos de VG, y permanencia en la relación de violencia. Se observa comprensión de algunos elementos de la VG, si bien también se ponen de manifiesto discursos posmachistas. Estos

${ }^{1}$ Recibido: 25/02/2021 Evaluado: 06/02/2021 Aceptado: 17/03/2021 
resultados exponen la necesidad de diseñar planes de estudio que incorporen el abordaje de la VG.

Palabras clave: Violencia de género, Estudiantes universitarias, Grupos focales.

\begin{abstract}
After Law 1/2004 on Comprehensive Protection Measures against Gender Violence (GBV) was approval, advances have been identified, but also transformations in some social discourses that hinder progress in equality between women and men (Lorente, 2007). On the one hand, research shows that the myths around GBV are still current (Bosch and Ferrer, 2012; Bosch, Ferrer and Navarro, 2013; Delegación del Gobierno contra la Violencia de Género, 2014). On the other hand, the University should include and promote gender equality and non-discrimination transversally in education, teaching and research. However, the incorporation of gender equality and non-discrimination in undergraduate education has had little impact and has been surface. This work shows the ideas that university students of the Degree in Psychology have about gender violence. Two focus groups were carried out in which fourteen university students participated. The results revolve around five categories: patriarchal system, concept, causes, and types of GBV, and permanence in the relationship of violence. An understanding of some elements of GBV is observed, although posmachista discourses are also revealed. These results expose the need to design study programs that incorporate the GBV approach.
\end{abstract}

Keywords: Gender violence, University students, Focus groups

\title{
Introducción
}

La ONU (1995) define la Violencia de Género (VG) como todo acto "que resulte, o pueda tener como resultado un daño físico, sexual o psicológico para la mujer, inclusive las amenazas de tales actos, la coacción o la privación arbitraria de libertad, tanto si se producen en la vida pública como en la privada”. En España, hasta la formulación de La Ley Orgánica 1/2004, de 28 de diciembre, de Medidas de Protección Integral contra la Violencia de Género, la violencia hacia las mujeres en el marco de las relaciones de pareja se invisibilizaba bajo el nombre de violencia doméstica (Comas-d'Argemir, 2014). Este concepto obviaba que las causas y estrategias de perpetración de la violencia hacia las mujeres devienen de la estructura social de dominación masculina (Bustelo, López y Platero, 2007), esto es, del sistema patriarcal. La Ley Orgánica 1/2004 define la VG "como manifestación de la discriminación, la situación de desigualdad y las relaciones de poder de los hombres sobre las mujeres, se ejerce sobre éstas por parte de quienes sean o hayan sido sus cónyuges o de quienes estén o hayan estado ligados a ellas por relaciones similares de afectividad, aun sin convivencia", y "comprende todo acto de violencia física y psicológica, incluidas las agresiones a la libertad sexual, las amenazas, las coacciones o la privación arbitraria de libertad" (p. 10). Con esta Ley se pasó, por tanto, de considerar la VG como una cuestión 
privada, a ser tratada como un problema social a abordar mediante la acción colectiva (de Miguel, 2005; Ferrer y Bosch, 2007).

En los años que suceden a la aprobación de la mencionada Ley se han producido avances a nivel legislativo y en términos de visibilización de problemáticas derivadas del sistema sexogénero. Sin embargo, también han ido emergiendo discursos posmachistas que obstaculizan los avances en igualdad entre mujeres y hombres (Lorente, 2007). Por ejemplo, hay quienes consideran que la VG es una forma que los hombres tienen de defenderse de las provocaciones de las mujeres, quienes la justifican por la desesperación que éstas les producen, o la consideran un fenómeno residual. En otras ocasiones, condenan esta violencia, a la vez que rechazan la idea de que mujeres y hombres puedan ser iguales. Entre ellas hay quienes justifican la VG, consideran que una mujer maltratada se merece serlo, o quienes piensan que ya no es un problema de la envergadura que en un tiempo anterior (Actis, de Prada y Pereda, 2011). Sin embargo, entre los hombres también están los que consideran que la VG es un producto del machismo que existe en la actualidad, que es denunciable, y que para superarla es necesario que los hombres se comprometan de forma activa. Del mismo modo, las mujeres también consideran que es un problema de relevancia y difícil de superar, que todas las mujeres pueden ser víctimas de VG pero que la amenaza no es fácil de identificar, y que deriva del sistema social patriarcal en el que se vive (Actis, de Prada y Pereda, 2011).

Por otro lado, se observa que, a pesar de que existe un rechazo a nivel general hacia la VG, parte de la ciudadanía sigue sin saber qué actitudes y comportamientos constituyen este tipo de violencia. De hecho, el $31 \%$ se muestra conforme con algunos indicadores de violencia en la pareja (p. ej., control y restricciones) (Delegación del Gobierno contra la Violencia de Género, 2014). Asimismo, en el imaginario colectivo siguen estando presentes los estereotipos y mitos en torno al perfil de víctimas y agresores (Bosch y Ferrer, 2012; Bosch, Ferrer y Navarro, 2013). Por ejemplo, el 38\% de la población tiende a clasificar a los agresores bajo alguna patología mental. A su vez, está presente el mito de los perfiles de mujeres víctimas, esto es, aquellas que pertenecen a grupos vulnerables son más fácilmente maltratadas (Heise y García-Moreno, 2003). En cuanto a la permanencia en la relación, el $89 \%$ de la población opina que es común que la mujer no termine con la situación de maltrato por la presencia de hijas/os en la pareja y el $67 \%$ refiere a la dependencia económica (Delegación del Gobierno contra la Violencia de Género, 2014).

En población joven de entre 15 y 29 años, se observa cierta resistencia al cambio hacia un paradigma igualitario. Si bien, la juventud piensa que la VG no se puede aceptar, ellas manifiestan un mayor rechazo. Asimismo, el control no es identificado como VG en un tercio de la juventud, siendo este colectivo el que más consiente este tipo de comportamientos (de Miguel Luken, 2015). Algunos estudios ponen de manifiesto que en la población joven y adultez emergente (personas de 18 a 30 años) existen percepciones sobre la VG que no corresponden con la definición de dicha violencia en la ley, llegando a tolerarla y justificarla (Bajo, 2020; Bonilla, Rivas y Vázquez, 2017). Asimismo, se identifica una asimilación del modelo de amor romántico en el alumnado universitario que se relaciona con la normalización de la violencia (Bosch y Ferrer, 2013). Alrededor del 30\% de las alumnas universitarias expresan haber sufrido alguna forma de maltrato en su relación de pareja (p. ej., violencia física, sexual, difusión de imágenes íntimas por internet) (Díaz-Aguado, 2013). 
Los datos mostrados ponen de relieve la necesidad de que la población adquiera un mayor conocimiento e información objetiva sobre la VG (de Miguel Luken, 2015). En este contexto, su abordaje cobra una alta importancia en las universidades, puesto que se erigen como institución fundamental en la formación y transmisión de valores (Castells, 2001). Sin embargo, la universidad ha heredado, de generación en generación, un modelo de relación que dificulta instaurar prácticas no sexistas que lleguen a legitimarse por la comunidad universitaria, ejerciendo sus propias estrategias discriminatorias (Martínez, Merma-Molina, y Ávalos, 2018; Morrison y Kelley, 2005). Mendivelso (2008) expone la responsabilidad de la academia en la educación de las nuevas generaciones, que a veces olvida que es transmisora de ética y responsabilidad social, preocupándose casi de modo exclusivo de la formación técnica. Además, hay autoras que ponen de manifiesto la importancia de que ciertas disciplinas se hagan responsables - desde la universidad — de las implicaciones de las violencias a nivel social (Vaca y Díaz, 2009).

A pesar de que el Capítulo II de la Ley 1/2004 - ya mencionada — contempla la garantía de integrar el principio de igualdad en la educación, en el ámbito universitario el objetivo de transversalizar la perspectiva de género en los planes de estudio ha tenido un bajo impacto y la incorporación, en la mayoría de casos, ha sido superficial (Guarinos y Cobo, 2018).

Este trabajo es una aproximación a los conocimientos sobre la VG de estudiantes universitarias de un Grado en Psicología. Se pretende constatar la necesidad de incluir formación en materia de $\mathrm{VG}$ en los planes de estudios universitarios para contribuir a su prevención, detección y actuación contra la misma (Canplloch, Alegre y Pérez, 2012). Se utilizó la metodología cualitativa con el objetivo atender a las voces implicadas en la definición y, así, orientar el diseño de la formación en base al punto de partida de las estudiantes.

\section{Objetivo general y preguntas de investigación}

El objetivo general de este estudio es conocer qué piensan las alumnas del Grado de Psicología sobre la VG. Para dar respuesta, se plantearon las siguientes preguntas de investigación: (1) ¿Qué entienden las estudiantes universitarias por violencia de género?; (2) ¿Qué tipos de violencia de género conocen?; (3) ¿Qué causas de la violencia de género identifican?; (4) ¿Qué explicaciones dan al mantenimiento de la relación de violencia?

\section{Método}

\section{Participantes}

Las participantes fueron 14 alumnas del Grado en Psicología que tenían entre 18 y 22 años. De las 14 participantes, tres estaban en primero curso, cuatro en segundo, cuatro en tercer curso y tres en cuarto curso del grado. Algunas se conocían entre sí, por ser compañeras de clase. Si bien, las participantes que no coincidían en el mismo curso no se conocían.

\section{Procedimiento}

Se realizaron dos grupos focales, participaron siete alumnas en cada uno. El muestreo se llevó a cabo a través de la estrategia bola de nieve. La selección de participantes se realizó con el 
objetivo de que el grupo fuera heterogéneo respecto a las ideas en torno a la VG y estuvieran representados todos los cursos del grado.

Las sesiones se grabaron en audio, bajo consentimiento informado de las participantes. Ambos grupos focales tuvieron una duración de entre 90 y 100 minutos. Las grabaciones fueron transcritas, se utilizaron seudónimos para garantizar el anonimato.

La dinamización de los grupos focales la llevó a cabo una profesora del Grado en Psicología. El rol de observadora lo desarrolló una alumna colaboradora, seleccionada por su grado de sensibilidad y conocimiento del tema a estudiar (Canplloch, Alegre y Pérez, 2012). El análisis de la información lo realizaron dos psicólogas que se identifican como feministas, con formación y experiencia en investigaciones-intervenciones en salud de las mujeres.

La recogida de la información se sitúa previa a la fecha del 8 de marzo de 2018, del movimiento Me Too, o el llamado caso de "La Manada", considerados hitos que han ido marcando la agenda de la concienciación sobre las reivindicaciones del movimiento feminista en los últimos años.

\section{Instrumento}

Se diseñó un guion de entrevista dividido en cuatro bloques temáticos que indagaban sobre las ideas y pensamientos en torno al concepto, tipología e identificación de las causas de la VG.

\section{Análisis}

Se adoptó una orientación experiencial del análisis temático (AT), centrada en lo que las participantes piensan, sienten y hacen. Se basa en la idea de que el lenguaje refleja la realidad (Braun, y Clarke, 2006). En función del nivel de interpretación suelen reconocerse tres niveles de códigos. En este trabajo se ha realizado un análisis descriptivo.

Los dos grupos focales se analizaron secuencialmente. El primero sirvió para explorar categorías emergentes. Se realizaron comparaciones constantes entre categorías y sus relaciones. La información obtenida se volvía a contrastar con la información inicial para precisar su definición. Los memos que fueron emergiendo en el proceso analítico contribuyeron a la reflexividad y a la interpretación de la información. Para el análisis de la información se empleó como apoyo el programa informático Atlas-ti. 8.0. Para garantizar la validez se realizaron comprobaciones de coherencia, facilitando las descripciones de las categorías elaboradas a otra codificadora.

\section{Resultados}

El análisis de los grupos focales mostró que, al preguntar a las estudiantes sobre VG, los resultados giran en torno a diversas ideas. Aluden al sistema patriarcal en la que se enmarca y que la mantiene, la definen y mencionan sus causas. Además, en las narrativas mencionan los tipos de VG e indican las razones que creen que dificultan la salida de la relación violenta. En la figura 1 se muestra el sistema de categorías obtenido del análisis. Sus elementos se detallan a continuación. 
Sistema patriarcal

Se alude al patriarcado como el sistema social en el que tiene lugar la VG. El debate gira en torno al sustento que proporciona a instituciones, y la sociedad en general, para que la VG permanezca a lo largo del tiempo. Además, piensan que en este sistema las mujeres víctimas de VG no encuentran el apoyo necesario para salir de la situación de violencia que viven.

se ve apoyado por instituciones y por la sociedad en si porque es una sociedad patriarcal, al fin y al cabo, yo creo que ese es el problema, en el momento en que esa sociedad y las instituciones no lo apoyaran serían erradicado (Susana)

la sociedad está respaldando eso, y las instituciones que se supone que tienen que defender a una mujer maltratada está respaldando eso también... (Susana)

Concepto de violencia de género

Tomando como base la direccionalidad de la violencia, la discusión en enmarca en torno a dos ideas:

Violencia hacia la mujer. Se incluyen en esta categoría las narrativas que alude a la definición de VG, entendiéndola como la manifestación de la asimetría de poder entre mujeres y hombres, y diferenciándola de la violencia doméstica.

primero dejar claro que es desde los hombres hacia las mujeres y no al revés, yo creo que eso tiene que quedar muy claro, porque el primer "cuñadismo" que te sueltan cuando hablan de violencia de género es "y las violencias de la mujer hacia los hombres", no es violencia de género. Yo considero que es eso, puede existir violencia doméstica [...] lo puede maltratar por muchos otros motivos, pero no por ese, entonces yo creo que es lo primero que hay dejar claro, por eso a mí me gusta más usar el término violencia machista, para dejar claro que es de los hombres a lass mujeres (Paloma)

se sienta con más poder el hombre sobre la mujer [...] el caso es que se sienta superior a ella (Marta) 


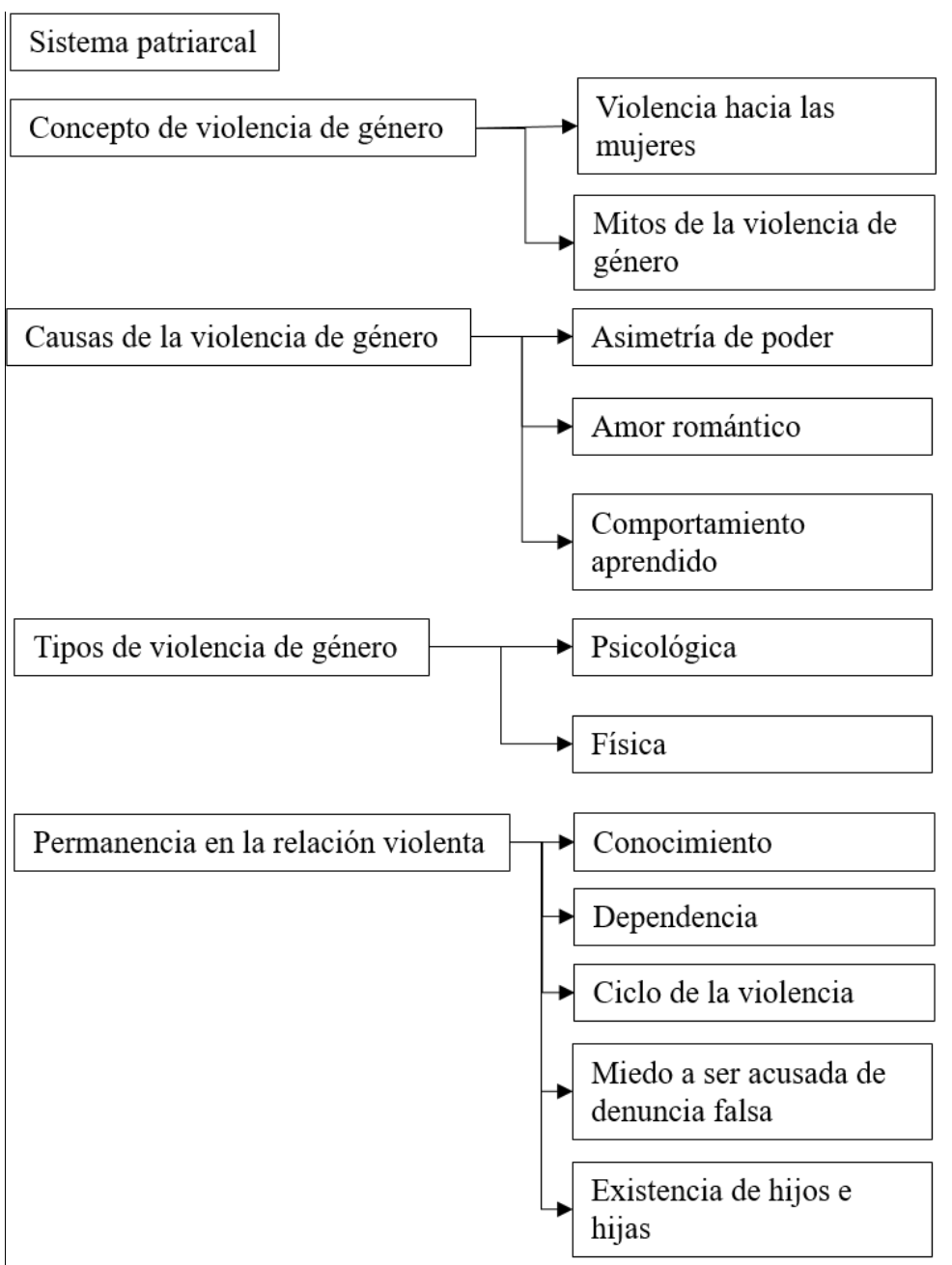

Figura 1. Sistema de categorías

Mitos de la violencia de género. Engloba aquélla citas en las que, si bien, las participantes no llegan a indicar que la VG sea aquélla que puede ejercer la mujer hacia el hombre, muestran la necesidad de hablar del fenómeno y de poner el énfasis en lo que entienden como su invisibilización.

todavía siguen estando un poco por encima nuestra, pero también creo que hay muchísimos hombres "tapados" por así decirlo que sufren mucha violencia por parte de las mujeres, más de lo que podemos llegar a ser conscientes (Nuria)

Yo creo que ellos mismos ni siquiera lo notifican por vergüenza (Alejandra)

Además, en las narrativas se observa la consideración de los hombres como grupo oprimido y la individualización de la VG, obviando que constituye un problema social.

los hombres también están sufriendo el machismo con opresión, lo que estáis hablando de la violencia ejercida de la mujer al hombre eso es opresión, porque ellos no salen en los medios de comunicación porque no denuncian, porque son los machos como van a dejar que una mujer le pegue, no puede ser (Marta) 
Más relevancia no, la misma. Muere una persona (Nuria)

Causas de la violencia de género

Preguntadas por las causas de la VG, las estudiantes mencionan que en su base están el poder, el amor romántico y el comportamiento aprendido.

Asimetría de poder. Las relaciones de poder asimétricas en las que el hombre tiene el rol dominante y la mujer el subordinado, es considerado uno de los motivos por los que tiene lugar la VG. Para el hombre, ostentar la posición de superioridad implica creer que tiene derecho a considerar y tratar a la mujer como un ser inferior a él, por ejemplo, mediante comportamientos y actitudes violentas.

si sigue existiendo esa superioridad del hombre sobre la mujer, va a seguir en todos los ámbitos, y si un hombre en un momento dado en su relación de pareja se siente superior a ella, cree que puede tener ese derecho digamos de hacerla sentir inferior, porque como él se ve superior, "yo tengo ese derecho de recordarte a ti que tú eres inferior" (Elena)

porque hay muchos hombres que todavía sienten la superioridad y creen que eso les da derecho a tratarnos como quieren (Alejandra)

Amor romántico. Las participantes expresan varias situaciones en las que se ponen de relieve los mitos del amor romántico y las posiciones subjetivas tradicionales de mujeres y hombres. Carmen se refiere al mito de la omnipotencia del amor, o amor fusional, cuando habla del "verdadero amor". Asimismo, apunta al mandato, interiorizado en las mujeres, del cuidado y satisfacción de necesidades del otro. Menciona que estas situaciones afectan principalmente a mujeres jóvenes, aludiéndose a la idea de la existencia de perfiles de víctimas.

en las relaciones así más de jóvenes y eso, cuando la mujer se siente, vamos tiene maltrato psicológico, pienso que también se debe un poco a que su pareja le hace sentir especial, en el sentido, de que solo ella puede ayudarle, puede apoyarle, solo ella conoce su verdadera yo, y se supone que si estás en pareja quieres al otro, ella se siente como en la responsabilidad u obligación de excusarle ante los demás, de defenderle, me trata así porque tiene esta historia detrás, yo le quiero y como yo sé cómo es, yo lo defiendo...(Carmen)

El mito de los celos como muestra de amor, pero también como síntoma de inseguridad, es considerado un elemento base de la VG.

pero ahora es que tenemos tanta variedad y esos celos es por miedo, la violencia que se tiene es por miedo al tener tantas posibilidades es porque te puedo perder tan fácilmente, que me sale por ahí. No estoy justificando, pero puede ser una de las causas, el miedo de por qué me vas a elegir a mí teniendo tantas posibilidades (Marta) 
Juana alude al mito de la media naranja al mencionar el tratamiento a la mujer como "princesa", a la "la pareja perfecta", o el "romanticismo". De nuevo se pone de manifiesto la creencia de que hay perfiles más proclives a sufrir VG.

el hombre a ti te trata como a una princesa, ¿no? Que se suele decir, y para fuera es la pareja perfecta, lo que pasa que poco a poco, porque ya es por un caso muy cercano mío, a ver una niña con estudios, que tú hablas con ella y en ningún momento tú vas a pensar que va a sufrir eso, pero ya una vez que te da cuenta de que estas ahí, estas tan enamoradas que lo permites ¿no?, porque es eso el romanticismo de "no si me lo ha pedido es porque me quiere, porque no quiere que otro se fije en mi” (Juana)

Comportamiento aprendido. Al mencionar la VG como comportamiento aprendido se está hablando de la transmisión intergeneracional de la VG, respecto a la cual se observan distintas posturas. Lola piensa que una de las causas de la $\mathrm{VG}$ es haberlo vivido en el seno del hogar familiar y que se ejerce como forma de reproducir las pautas de relaciones y patrones conductuales machistas aprendidos.

han aprendido en sus casas creo yo, porque eso se da sobre todo ahora mismo en personas más mayores, entonces si por ejemplo un hombre con cincuenta años ha vivido que su padre le pegaba a su madre, él coge ese comportamiento y lo lleva a su casa, y si tiene hijos ellos lo ven como normal y cuando se case y tenga hijos lo va a seguir haciendo... (Lola)

Sin embargo, Alejandra apunta a que puede haber otros factores que hacen que los hombres ejerzan VG.

pero yo no creo que todos los hombres que maltraten lo hayan aprendido en sus casas (Alejandra)

\section{Tipos de $V G$}

Las participantes distinguen dos tipos de VG, psicológica y física:

Violencia psicológica. El debate gira en torno a varias cuestiones. Por un lado, a su "aceptación" social y falta de reconocimiento. Por otro, a sus consecuencias a nivel personal, que concretan en el daño que ocasiona en la autoestima. Marta considera que es preciso el amor propio para que otras personas puedan quererte.

Yo creo que eso también se ve a "puñao", tanto por la sociedad, como por instituciones como por muchísima gente, que a lo mejor ven mal que tú le des una paliza pero lo que es la violencia psicológica primero que la mayoría de las personas ni siquiera la reconocen como violencia (Susana)

o por lo que dijo antes ella, la violencia psicológica va devorando la autoestima $y$, al final, si no te quieres a ti quién te va a querer (Marta) 
El control a través de las TRICs es considerado como una forma concreta de violencia psicológica que conecta con los celos.

Es muy tonto, si es que por ejemplo ahora con Instagram, que sale que puedes ver quién le ha dado me gusta a una foto, y si tú tienes una novia o un novio y ves que una novia por ejemplo le ha dado me gusta a una foto un tío, es como madre mía le ha dado me gusta a la foto de otro y te montas una paranoia, que es que le has dado dos veces a una pantalla de un móvil... (Nuria)

Violencia física. Susana y Juana ponen como ejemplos de violencia física el pegar, dar palizas, o la muerte.

y ya te está maltratando, te pega una paliza de muerte (Susana)

claro por qué en el momento en que se enfada te pega, ya ven el tipo de violencia que sea (Juana)

Permanencia en la relación de violencia

La permanencia en la relación de violencia la explican a través de cinco factores: conocimiento sobre el fenómeno - aspecto que genera opiniones diversas - , la dependencia, el ciclo de la violencia, el miedo a ser acusada de poner una denuncia falsa y la existencia de hijas e hijos.

Conocimiento. Para Lola el conocimiento sobre el fenómeno es clave para iniciar y mantenerse en una relación violenta. Consideran que ser feminista te hace detectar más fácilmente los indicadores de VG, aunque finalmente, no exime sufrirla.

una mujer que es capaz de ser decidida e independiente ella sola, antes de que llegue a las manos, sabe dejar a ese hombre. Si algún día en plan a los dos meses está viendo que la controla, porque una mujer que es feminista, que sabe lo que tiene que hacer, que a lo mejor está siendo controlada... una mujer que sabe que eso está mal y lo va a dejar (Lola)

Paloma y Susana ponen el énfasis en factores emocionales y en el modo en que evoluciona la VG. Consideran que identificarse como feminista no evita ser víctima por cómo la violencia se va instaurando paulatinamente — sin que la víctima sea consciente-, ejerciéndose primero violencia psicológica. Se distingue entre razón y sentimientos, mostrándose cómo estos, a veces, se revelan contradictorios, facilitando el inicio y mantenimiento de la VG.

conozco mujeres feministas que han sufrido violencia de género, porque incluso tu siendo feminista y siendo consciente e identificando todos los patrones eres... puedes ser víctima perfectamente, porque puede generar esa dependencia emocional (Paloma)

esto de aquí no funciona al mismo tiempo de la razón, es que por mucho que tú digas "esto está mal, esto está mal, esto está mal", la dependencia emocional y el

No 9, 2021. Página | 169 
maltrato psicológico que lleva ocurriendo durante equis tiempo acaba haciéndote que puedas ser víctima y estés en esa situación muchísimo tiempo (Susana)

Dependencia. Consideran la dependencia emocional y económica los motivos por los que las mujeres permanecen en la relación de violencia. De alguna forma, una dependencia que impide ver otras opciones vitales. Se mencionan también a la familia y los hijos e hijas, aspecto éste al que se aludirá más adelante.

dependencia emocional... más allá de la económica, y que muchas veces económica, y después cuestión de la familia, los niños... (Paloma)

Ciclo de la Violencia. Las participantes refieren indirectamente a las fases del ciclo de la VG. Se inicia con la violencia psicológica y, progresivamente-hecho que Carmen ejemplifica con la metáfora de la rana-, va evolucionando hacia violencia física. Se menciona la dificultad para identificar que se está en una situación de violencia.

no se va a ir a ti y te va a dar un guantazo de repente, empieza psicológicamente y por muy fuerte mentalmente que tú seas... acabas cayendo (Susana)

quería compartir una metáfora que yo leí una vez y era de una rana que decía: "si tú metes una rana en un cazo de agua hirviendo la rana salta, pero si metes a una rana en un cazo de agua y le vas dando temperatura poco a poco hasta que alcanza la temperatura del cazo anterior, y no salta sabes". Es hoy te doy un poquito de violencia pero te trato genial, y poco a poco estás hundida y no sabes cómo salir de ahí y hay veces que la persona no sabe que está quemándose (Carmen)

En la cita anterior, Carmen ya hacía referencia al reforzamiento intermitente que tiene lugar en el ciclo de la violencia, al transitar de las fases de acumulación de la tensión y estallido de la violencia, a la de arrepentimiento, que actúa como reforzamiento positivo. Susana expresa que esta pauta repetida a lo largo del tiempo, por una persona a la que la mujer siente querer, hace que pueda normalizarse la situación.

esa dependencia emocional de por medio, al día siguiente probablemente antes de volverte a maltratarte psicológicamente te está pidiendo perdón, y tu sientes algo por esa persona, y una dependencia emocional muy grande, te está pidiendo perdón es plan "se ha arrepentido", a la siguiente vez te lo vuelve a hacer y te vuelve a pedir perdón, tu acabas viéndolo normal (Susana)

que no lo está haciendo porque sea malo o porque sea "ná", sino porque me quiere (Juana)

Paloma expresa cómo, a lo largo del ciclo de la violencia, la mujer va haciéndose más depedendiente, mientras el agresor aprende que, tras cada agresión, logra mantener el poder.

pero en la mayoría de los casos el hombre es capaz de hacer que la mujer depende de él emocionalmente (Paloma) 
Miedo a ser acusada de denuncia falsa. El miedo a ser acusada de denuncia falsa y a tener que volver con el agresor se señala como factor clave para el mantenimiento en la relación de violencia. Susana apunta que esto tiene lugar por el temor a la falta de apoyo a nivel institucional, social y familiar.

te pega una paliza de muerte sabiendo que lo has denunciado, y es lo que pasa en muchísimos casos, cuántos casos se denuncia y la denuncia ni prospera, ni llega a ningún sitio ni nada, y era real. Porque como hay denuncias falsas, hay una denuncia falsa pues ya está, todas las demás son denuncias falsas. Y no es así, y ese miedo de que la institución no te apoye, ni la institución, ni la sociedad, ni tu propia familia muchísimas veces haces que tu sigas en esa situación y digas "mira si digo algo puedo estar incluso peor" (Susana)

Existencia de hijas e hijos. La relación entre la existencia de hijas e hijos y la permanencia en la relación es considerada de dos modos. Por un lado, como una circunstancia que dificulta la ruptura de la relación, agravando la situación. Consideran que la mujer sentirá que no debe separar a las hijas e hijos de su padre, y se obvia que, cuando se maltrata a la mujer, se ejerce maltrato hacia aquéllos.

una vez que tiene hijos y la chica es maltratada, como que es peor, la situación se agrava... ella se ve más dependiente de la persona porque son los padres de sus hijos... piensan que cómo van a separar a los hijos de su padre, y porque a lo mejor el maltrato es solo a ella, ya en un momento que a lo mejor que el maltrato va a hacia ellos también si lo ven de forma más clara, pero si es hacia ella nada más hay muchas mujeres que callan y aguantan por sus hijos (Juana)

Por su parte, Isabel considera que una mujer con hijas y/o hijos se separaría antes, precisamente por ellas y/o ellos.

creo que la mujer que decide separarse de su marido, de su pareja que tiene hijos, lo hace por sus hijos, no lo hace realmente por ella, yo creo que es más complicado que una mujer que es maltrata por un hombre sin tener hijos se separe antes que una mujer con hijos (Isabel)

\section{Discusión}

Algunas participantes expresan que la VG se trata de un fenómeno estructural que tiene lugar en un sistema patriarcal y que se dirige contra las mujeres. Destacan el papel de las instituciones que, por sus estructuras patriarcales heredadas, no brindan toda la protección necesaria a las mujeres, contribuyendo a su perpetuación (Expósito, 2011). Esta idea es consonante con el concepto de patriarcado como "la toma de poder histórica por parte de los hombres sobre las mujeres cuyo agente ocasional fue de orden biológico, si bien elevado éste a la categoría política y económica. Dicha toma de poder pasa forzosamente por el sometimiento de las mujeres a la maternidad, a la represión de la sexualidad femenina, y a la apropiación de la fuerza de trabajo total del grupo dominado, del cual su primer, pero no único producto son los hijos" (Sau, 1981/2000, pp., 237-238). 
Hay participantes que identifican la VG como aquélla que se produce contra la mujer, por el hecho de serlo, y en base a la asimetría de poder entre mujeres y hombres. Esto es coincidente con las definiciones del fenómeno, que la entienden como aquélla ejercida contra las mujeres por su pareja o expareja varón a través de un patrón de comportamiento habitual. Su objetivo es ejercer control y poder sobre la relación y la pareja sentimental (Bosch, Ferrer, Ferreiro, y Navarro, 2013). En contraposición, se identifican ciertas ideas consonantes con el posmachismo (Lorente, 2009) cuando se observa la necesidad de hacer referencia, comparar, o llegar a igualarla con la violencia que puedan experimentar los hombres por parte de sus parejas mujeres. En esta línea, indican que la importancia del fenómeno no reside en la cantidad de víctimas, para poner de relieve lo que consideran la invisibilización del fenómeno, o poner de manifiesto que, al menos en lo que refiere a la VG, los hombres son un grupo oprimido por no considerarse víctimas de la misma. Se obvia, por tanto, el carácter estructural de la VG-individualizando el fenómeno-, y del concepto de opresión, que atiende a las relaciones jerárquicas - con frecuencia basadas en características fijadas en el nacimiento o fuera del control personal-, anclada en sistemas opresivos con raíces históricas (Freire, 1970/2009; Prilleltensky, 2008; Prilleltensky y Gonick, 1994).

En este sentido, alguna participante propone dar un giro en la denominación de VG, por la posible confusión que puede generar, y pasar a utilizar el término "violencia machista". Este concepto ya queda recogido en la Ley 7/2018, de 30 de julio, por la que se modifica la Ley 13/2007, de 26 de noviembre de medidas de prevención y protección integral contra la violencia de género, aprobada por el Parlamento de Andalucía.

Las participantes identifican diversas causas de la VG. Por un lado, la asimetría de poder entre mujeres y hombres, caracterizada por la dominación masculina (Bourdieu, 2000; Daune-Richard y Hurtig, 1995). Además, mencionan los mitos del amor romántico (Luzón, Ramos, Recio y de la Peña, 2011). Por un lado, consideran los celos como causa de la VG, obviándose que constituyen una de las técnicas usadas por los maltratadores para ejercer control sobre las mujeres, especialmente sobre sus redes sociales. El mito de la omnipotencia, o creencia en el amor fusional, puede usarse para eludir ciertos cambios comportamentales o actitudinales que restan importancia a los conflictos de pareja, obstaculizando su afrontamiento e implican el anclaje en la idea de la esperanza del cambio por amor. Por su parte, el mito de la media naranja puede llevar a un alto nivel de tolerancia hacia comportamientos dañinos, al creer que al ser la pareja ideal hay que permitirle más a la otra persona y una misma tiene que esforzarse más para que la relación funcione (Bosch, Ferrer, Ferreiro y Navarro, 2013). Como puede observarse, estas ideas están presentes en las narrativas de las participantes y en lo que consideran elementos base para la VG. Además, enlazan con la construcción del ideal femenino en el que el amor y el ser-para-otros se constituyen como elementos centrales y el sentido de la vida (Expósito, 2011; Muruaga y Pascual, 2013; Velasco, 2009).

Algunas narrativas reflejan la idea de que la VG se aprende en la familia de origen, es la llamada transmisión intergeneracional de la VG. Sin embargo, los datos apuntan a que entre el $15-30 \%$ de los hombres que han sido víctima o testigo de maltrato en la infancia, lo ejercerían posteriormente. Se estaría hablando de un factor de riesgo más que causal (Ehrensaft, et al., 2003; Gago, 2006; García Moreno, 2005). Este constituye uno de los mitos de la VG asociados a los maltratadores. 
En relación a los tipos de $\mathrm{VG}$, los discursos giran en torno a la violencia psicológica y física, considerándose la primera necesaria para que tenga lugar la segunda. Sin embargo, no se mencionan otros tipos de violencia, como la sexual, económica, espiritual o social. Tampoco se hace referencia a los distintos modelos explicativos de la VG (Bosch, Ferrer, Alzamora, 2006; Bosch, Ferrer, Ferreiro y Navarro, 2013).

El mantenimiento en la relación violenta lo explican por diversas razones: el conocimiento del fenómeno, identificarse como feminista, y la juventud. De esta forma, las participantes tratan de identificar perfiles de mujeres proclives a experimentar VG. Si bien, el maltrato puede ocurrirle a cualquier mujer, con independencia del país de procedencia, clase social, etnia, raza, religión, nivel educativo, etc. La existencia de perfiles de víctimas es uno de los mitos que existen en torno a las víctimas de VG (Bosch, Ferrer, Ferreiro y Navarro, 2013; Gago, 2006; García Moreno, 2005).

La dependencia emocional y económica se mencionan como elementos que dificultan la salida de la relación violenta (Basu y Famoye, 2004; Bhattacharjee y Banda, 2016). Por un lado, señalan el impacto de la VG en la posibilidad de vislumbrar otras opciones vitales, debido al deterioro en la autoestima y en la percepción de autoeficacia. Esto contribuye a que la mujer no se sienta capaz de emprender la ruptura de pareja y un nuevo proyecto vital con ciertas garantías de éxito. Por otro lado, la dependencia emocional se ve reforzada por el control ejercido sobre la red social que tiene lugar a lo largo del proceso de VG, aislando a la mujer de su red de apoyo.

Sin nombrarlo, las participantes van haciendo referencia a distintas fases del ciclo de la VG y a los mecanismos que los sustentan. En este sentido, sus alusiones al inicio progresivo a través de la violencia psicológica, a las conductas de arrepentimiento que lleva a cabo el maltratador, y al impacto que tiene en la mujer el reforzamiento intermitente que implica el ciclo de la VG, es consonante con la literatura existente (Expósito, 2011; Walker, 1979). Sin embargo, el ciclo de la VG también se caracteriza por unas posiciones subjetivas en mujeres y hombres y que explican su dinámica (Velasco, 2009). Este elemento está ausente en las narrativas de las participantes.

Las alusiones al miedo a ser acusadas de denuncia falsa es un efecto, entre otros, del posmachismo (Lorente, 2009). Éste va introduciéndose en el discurso social y generando una situación de indefensión y miedo en las mujeres. A su vez, invisibiliza el dato de que, por ejemplo, de las 1.557.190 denuncias que se han registrado desde 2009, se han incoado 121 procedimiento por denuncia falsa, de los cuales únicamente el 0,0069\% han terminado con una sentencia condenatoria (Fiscalía General del Estado, 2020). Asimismo, el miedo también hace referencia a tener que volver con el maltratador, al no facilitárseles los recursos necesarios para salir de la relación de violencia. Esto se vincula con las ideas comentadas en torno al sistema patriarcal y a la falta de una acción determinada para erradicar la VG.

\section{Conclusiones}

A través de estos resultados se evidencia que las participantes coinciden en que la VG se presenta como un fenómeno que abarca todos los niveles de la sociedad. Sin embargo, no 
todas la consideran como una forma de violencia de origen sistémico. Esto se explicaría al detectar mitos e ideas posmachistas, como la necesidad de aludir al hombre como víctima cuando se está debatiendo en torno a la VG. Este discurso consigue generar confusión en los conceptos, a la vez que se muestran conscientes del impacto negativo que puede tener en las víctimas el discurso de las denuncias falsas. Asimismo, se observan limitaciones al reconocer las causas de la VG. Si bien, hay acuerdo en considerar la asimetría de poder, o los mitos amor romántico como elementos centrales, hay posturas encontradas en cuanto a su aprendizaje en el seno de la familia de origen-lo que constituye un mito de la VG-. Por su parte, no se hace referencia a ningún modelo explicativo de la VG. Las limitaciones en el discurso se manifiestan también al hacer referencia a dos tipos de VG, la física y la psicológica, obviándose, por ejemplo, otras formas como la económica o espiritual. La diversidad de opiniones también tiene lugar en torno a la existencia de perfiles de víctimasde nuevo un mito sobre la VG-, y en torno al rol de hijas e hijos al facilitar o dificultar la salida de la VG. En general, se observa que, a pesar de los avances, los discursos de las participantes reflejan creencias y mitos sobre la VG y no un conocimiento fundamentado.

Una de las limitaciones de este trabajo es que se presentan resultados de una parte de la muestra. Asimismo, el hecho de que parte de las estudiantes se conocieran, y también conocieran a la profesora que dinamizó los grupos focales, puede haber condicionado las respuestas a las preguntas y del desarrollo del grupo.

Para dar respuesta a la situación descrita se propone incorporar formación específica en VG en los estudios universitarios que carezcan de ella. Especialmente en aquellos enmarcados en ciencias de la salud y ciencias sociales. Algunos de los contenidos a incluir podrían ser los modelos explicativos sobre la VG, el significado de sistema patriarcal, el ciclo de la VGincluyendo las posiciones subjetivas que adoptan tanto hombres como mujeres-, o los tipos de VG. Se considera así que la institución universitaria dará un paso más para, por un lado, asumir la responsabilidad social que le corresponde en la erradicación de la VG y, por otro, para proporcionar una formación más completa a las y los profesionales que deben hacer frente a este problema social.

\section{Agradecimientos}

Se agradece la participación de las alumnas del Grado de Psicología.

\section{Referencias}

Actis, W., de Prada, M.A., y Pereda, C. (2011). Actitudes de la población ante la violencia de género en España. Ministerio de sanidad, política social e igualdad. Centro de publicaciones.

Bajo, I. (2020). La normalización de la violencia de género en la adultez emergente a través del mito del amor romántico. Cuestiones de género: de la igualdad y la diferencia, (15), 253-268.

Basu, B., y Famoye, F. (2004). Domestic violence against women, and their economic dependence: a count data analysis. Review of Political Economy, 16(4), 457-472. 
Bhattacharjee, A., y Banda, S. (2016). Domestic violence against women: The socioeconomic scenario. Indian Journal of Health and Wellbeing, 7(7),765-768.

Bonilla, E., Rivas, E., y Vázquez, J.J. (2017). Tolerancia y justificación de la violencia en relaciones de pareja adolescentes. Apuntes de Psicología, 35(1), 55-61.

Bosch, E., y Ferrer, V. A. (2012). Nuevo mapa de los mitos sobre la violencia de género en el siglo XXI. Psicothema, 24(4), 548-554.

Bosch, E., Ferrer, V.A., y Alzamora, A. (2006). El laberinto patriarcal. Reflexiones teóricoprácticas sobre la violencia contra las mujeres. Barcelona: Anthropos Editorial.

Bosch, E. Ferrer, V.A., Ferreiro, V. y Navarro, C. (2013). La violencia contra las mujeres. El amor como coartada. Barcelona: Anthropos Editorial. Grupo Editorial Siglo Veitinuno.

Braun, V. y Clarke, V. (2006). Using thematic analysis in psychology. Qualitative research in Psychology, 3(2), 77-101.

Bustelo, M., López, S. y Platero, R. (2007). La representación de la violencia contra las mujeres como un asunto de género y un problema público en España, en Bustelo, M. y Lombardo, E. (Eds.), Políticas de igualdad en España y en Europa. Madrid: Cátedra, 67- 96.

Canplloch, M., Alegre, R. M., y Pérez, M. D. (2012). Luces y sombras en la formación sobre prevención y violencia de género: valoración y percepción del profesorado, estudiantado y movimientos sociales. Revista interuniversitaria de formación del profesorado, 26(1), 57-74.

Comas-d'Argemir, D. (2014). La violencia de género en los medios de comunicación. Cómo y cuándo se representa como un problema público. En Actas del XIII Congreso de Antropología de la Federación de Asociaciones de Antropología del Estado Español, Periferias, fronteras y diálogos.

Daune-Richard, A. M. y Hurtig, M. C. (1995). Catégories et représentations de sexe: Un débat loin d'etre clos. En Ephesia (Ed.), La place des femmes: Les enjeux de l'identité et de l'égalité au regard des sciences (pp. 426-438). Paris, Francia: La Découverte.

De Miguel, A. (2005). La construcción de un marco feminista de interpretación: la violencia de género. Cuadernos De Trabajo Social, 18, 231-248. Recuperado de https://cutt.ly/oz6Yurd

De Miguel Luken, M. (2015). Percepción de la Violencia de Género en la Adolescencia y la Juventud. Delegación del Gobierno para la Violencia de Género. Ministerio de Sanidad, Política Social e Igualdad. Centro de Publicaciones. 20:1-243. ISBN: 97884-7670-728-9. 
Delegación del Gobierno contra la Violencia de Género (2014). Percepción Social de la Violencia de Género. Recuperado de https://n9.cl/06emc

Díaz-Aguado, M.J. (Coord.) (2013). La Juventud Universitaria ante la Igualdad y la Violencia de Género. Universidad Complutense de Madrid: Delegación del Gobierno para la Violencia de Género.

Ehrensaft, M., Cohen, P., Brown, J., Smailes, E., Chen, H. y Johnson, J. (2003). Intergenerational transmission of partner violence: A 20-year prospective study. Journal of Consulting and Clinical Psychology, 71, 741- 753.

Expósito, F. (2011). Violencia de género. Mente y Cerebro, Mayo/Junio. Recuperado de https://n9.cl/gh39h

Ferrer, V., y Bosch, E. (2007). El papel del movimiento feminista en la consideración social de la violencia contra las mujeres: el caso de España. Labrys $n^{\circ} 10$. Dossier España. Recuperado de https://cutt.ly/Lz6T11W

Ferrer, V., y Bosch, E. (2013). Del amor romántico a la violencia de género. Para una coeducación emocional en la agenda educativa. Revista de Currículum y Formación de Profesorado, 17(1), 105-122.

Fiscalía General del Estado (2020). Memoria de la Fiscalía General del Estado 2020. Recuperado de https://cutt.ly/uz6YULp

Freire, P. (2009). Pedagogía del oprimido. Madrid: Siglo XXI (Trabajo original publicado en 1970).

Gago, C. (2006). Atlas de las mujeres en el desarrollo del mundo. Madrid: Editorial SM.

García-Moreno, C. (Coord.) (2005). Estudio multipaís de la OMS sobre salud de la mujer y violencia doméstica. Primeros resultados sobre prevalencia, eventos relativos a la salud y respuestas de las mujeres a dicha violencia. Ginebra: OMS.

Guarinos, V., Caro González, F. J., y Cobo Durán, S. (2018). La igualdad de género en los estudios de grado en comunicación: la transversalidad imaginaria. Estudio de caso de las universidades públicas andaluzas. Revista Prisma Social, 296-235.

Heise, L.L., y García-Moreno, C. (2003). La violencia en la pareja. En E.G. Krug, L.L. Dahlberg, K.A. Mercy, A.B. Zwi y R. Lozano (Eds.), Informe Mundial sobre Violencia y Salud (pp. 97-131). Washington DC: Organización Panamericana de la Salud (Orig. OMS, 2002).

Ley Orgánica 1/2004, de 28 de diciembre, de Medidas de Protección Integral contra la Violencia de Género. (2004). Boletín Oficial del Estado, 313, de 29 de diciembre. 
Lorente, M. (2009). Los nuevos hombres nuevos. Barcelona: Destino.

Luzón, J. M., Ramos, E., Recio, P., y de la Peña, E. M. (2011). Factores de riesgo y de protección en la prevención contra la violencia de género en la pareja. Un estudio de investigación en la población adolescente andaluza. Recuperado de https://cutt.ly/sz6TGC7

Martínez, M.Á., Merma-Molina, G., y Ávalos, M.A. (2018). La brecha de género en la academia universitaria: inequidad de oportunidades de participación, capacitación y promoción. Momento: diálogos em educação, 28(3), 178-191.

Mendivelso, N. (2008). Ciencia y Ética: Matrimonio necesario para este milenio. Un Periódico, 118, 7.

Montero, M. (2003). Teoría y Práctica de la Psicología Comunitaria. Buenos Aires: Paidós

Morrison, Z., Bourke, M., y Kelley, C. (2005). Stop making it such a big issue: Perceptions and experiences of gender inequality by undergraduates at a British University. Women's Studies International ForuMorrm, 28, 150-162.

Muruaga, S., y Pastor, P. (2013). La salud mental de las mujeres. La psicoterapia de equidad feminista. Madrid: AMS, Asociación Mujeres para la Salud.

ONU (1995). Cuarta Conferencia Mundial sobre la Mujer. Beijing, Naciones Unidas, p. 214.

Prilleltensky, I. (2003). Understanding, resisting, and overcoming oppression: Toward psychopolitical validity. American Journal of Community Psychology, 31, 195-201.

Prilleltensky,basu I., y Gonick, L. (1994). The discourse of oppression in the social sciences: Past, present, and future. En E.J. Tricket, R.J. Watts, y D. Birman (Eds.), Human diversity: Perspectives on people in context (pp- 145-177). San Francisco: JosseyBass.

Sau, V. (2000). Diccionario ideológico feminista. Vol. I. Barcelona: Icaria. (Trabajo original publicado en 1981).

Vaca, P. V., y Díaz, M. C. R. (2009). Responsabilidad social de la Psicología frente a la violencia. Pensamiento Psicológico, 6(13), 87-96.

Velasco, S. (2009). Sexo, género y salud. Teoría y método para la práctica clínica y programas de salud. Madrid: Minerva Ediciones.

Walker, L. (1984). The battered woman syndrome. Nueva York: Springer. 